\title{
Analytical and Empirical Analysis of Countermeasures to Traffic Analysis Attacks
}

\author{
Xinwen Fu, Bryan Graham, Dong Xuan, Riccardo Bettati and Wei Zhao*
}

\begin{abstract}
This paper studies countermeasures to traffic analysis attacks. A common strategy for such countermeasures is traffic padding. We consider systems where payload traffic may be padded to have either constant inter-arrival times or variable inter-arrival times for their packets. The adversary applies statistical recognition techniques to detect the payload traffic rates and may use statistical measures, such as sample mean, sample variance, or sample entropy, to perform such a detection. We evaluate quantitatively the ability of the adversary to make a correct detection. We derive closed-form formulas for the detection rate based on analytical models we establish. Extensive experiments were carried out to validate the system performance predicted by the analytical method. Based on the systematic evaluations, we develop design guidelines that allow a manager to properly configure a system in order to minimize the detection rate.
\end{abstract}

\section{Introduction}

A significant portion of the Internet traffic today is encrypted, and there are strong indications that this portion will increase at a high rate. However, encryption alone may not be sufficient for secured communications. A number of non-cryptographic attacks $([9,13,18,21,22])$ have illustrated how simple observations of traffic behavior allow an adversary to infer significant information about participants and their communications. In an extreme case [21], it has been shown that timing analysis of SSH traffic can greatly simplify the breaking of passwords. This paper deals with timing based traffic analysis attacks and their countermeasures.

Traffic padding is one effective approach in countering traffic analysis attacks. The idea is based on Shannon's perfect secrecy theory: if one can map any payload traffic to a predefined pattern (a sufficient condition used by most researchers), then the adversary cannot obtain any information by analyzing the padded traffic. While in theory this technique sounds extremely simple, in reality, a perfect mapping cannot be achieved due to uncontrollable disturbances in a system. The

\footnotetext{
${ }^{*}$ Xinwen Fu, Bryan Graham, Riccardo Bettati and Wei Zhao are with the Department of of Computer Science, Texas A\&M University, College Station, TX 77843 - 3112 USA (E-mail:\{xinwenfu, bwg7173, bettati, zhao\}@cs.tamu.edu). Dong Xuan is with the Department of Computer and Information Science, Ohio State University, 395 Dreese Hall, 2015 Neil Ave., Columbus, OH 43210-1277 USA (E-mail:xuan@ cis.ohio-state.edu)
} 
question is: Do these disturbances result in information leaking, thus preventing a perfect secrecy system? If the answer is positive, metrics must be defined to assess the effectiveness of a particular implementation. In this paper, we propose using detection rate - defined as the probability that an adversary can make a correct identification of payload traffic rates - as the security metric.

Differing from the previous studies, we establish a formal theoretical framework for traffic padding systems. Based on this theoretical framework, we derive closed-form formulae for estimation of detection rates. Our formulae correctly reveal the relationship between detection rate and system parameters such as the padded traffic type, the sample size, and the location in the network where the adversary can collect traffic samples. We report results from extensive experiments in various situations including local area network in a laboratory, campus networks, and wide area networks. Our data consistently demonstrates the usefulness of our formal model and correctness of performance data predicted by the closed-form formulae. Based on the observations we make, we develop design guidelines that allow a manager to properly configure a system in order to minimize the detection rate.

The rest of this paper is organized as follows. Section 2 briefly reviews the related work and summarizes that Shannon's perfect secrecy theorem is the theoretical foundation in developing countermeasures to traffic analysis attacks. We present the network model, padding mechanism, and adversary strategy in Section 3. In Section 4 we develop a theoretical model and derive closed-form formulae for detection rates. Section 5 validates our theory by experiments. Section 6 summarizes this paper and discusses possible extensions.

\section{Related Work}

Shannon in [19] describes his perfect secrecy theory that is the foundation for the ideal countermeasure system against traffic analysis attacks.

The study of traffic analysis and its countermeasures for computer networks is not new. Baran [2] proposed the use of heavy unclassified traffic to interfere with the adversary's tampering on the links of a security network system for classified communication. He also suggested adding dummy, i.e. fraudulent, traffic between fictitious users of the system to conceal traffic loads.

To protect the anonymity of email transmission, Chaum [5] proposed the use of a Mix - a computer proxy. One technique used by a Mix is to batch and shuffle packets as they are forwarded; it has to collect a predefined number $K$ of fixed-size message packets from different users, shuffle the order of those packets, and then send them out. The reality is that a mix cannot always get $K$ packets efficiently from the users. So it is suggested that users send dummy messages of random and meaningless content to maintain a Mix's security and efficiency. Most researchers have suggested constant rate padding between the user and the proxy, e.g., [23]. Constant rate padding is also used here for preventing packet counting attacks [18].

A survey of countermeasures for traffic analysis is given in [28]. To mask the frequency, length, and origin-destination patterns of end-to-end communication, the use of dummy messages is suggested to make the traffic adhere to a predefined

pattern. From the discussion of Shannon's perfect secrecy theory, it is evident that a predefined pattern is sufficient but not 
necessary.

The authors in $([15,16,27])$ give a mathematical framework to optimize the bandwidth usage while preventing traffic analysis of the end-to-end traffic rates. Timmerman [26] proposes an adaptive traffic masking (hiding) model to reduce the overhead caused by traffic padding. But, when the rate of real traffic is low, the traffic padding rate is reduced as well, in order to conserve link bandwidth. Perfect secrecy is violated in this case, as large-scale variations in traffic rates become observable.

Raymond in [18] gives an informal survey of many ad hoc traffic analysis attacks on systems providing anonymous service and possible solutions. One conclusion is that dummy messages must be used to achieve high information assurance for the system. It is even claimed [1] that we have to use padding to each link of an anonymity network (although more research is needed to clear this claim).

In our previous work, NetCamo [12], we describe how to provide end-to-end prevention of traffic analysis while at the same time guaranteeing QoS (the worst case delay of message flows). It turns out that the delay experienced by packets of a protected flow is tightly coupled to the bandwidth required to send both payload and dummy packets. We propose methods such as QoS routing to tackle the QoS problem for systems using traffic padding strategies.

\section{The System Model}

In this section, we present the model of the network in which traffic analysis attacks may occur and then discuss traffic padding mechanisms that are used as a countermeasure for traffic analysis attacks. Finally we formally define the model of the adversary, who uses statistical pattern recognition strategies in traffic analysis attacks.

\subsection{Network Model}

In this work, we assume that the network consists of protected subnets, which are interconnected by unprotected networks. Traffic within protected subnets is assumed to be shielded from observers. Unprotected network can be public networks (e.g., the Internet), or networks that are deployed over an easily accessible broadcast medium. These networks are accessible to observation by third-parties, and are therefore open to traffic analysis. This model captures a variety of situations, ranging from battleship convoys (where the large-scale shipboard networks are protected and the inter-ship communication is wireless) to communicating PDAs (where the protected networks consist of single nodes).

Figure 1 illustrates the setup of the network we consider in this study. Protection from traffic analysis is provided by the two security gateways GW1 and GW2, which are placed at the two boundaries of the unprotected network and provide the traffic padding necessary to prevent traffic analysis of the payload traffic exchanged between the protected subnets A and B.

We note that the gateways can be realized either as stand-alone boxes, modules on routers or switches, software additions to network stacks, or device drivers at the end hosts. In this paper, we assume that they are stand-alone boxes. Nevertheless, the analysis in this paper is also effective for other implementations. To simplify the discussion, the communication is one way from Subnet A to Subnet B. Consequently, GW1 and GW2 are also called sender gateway and receiver gateway respectively. 


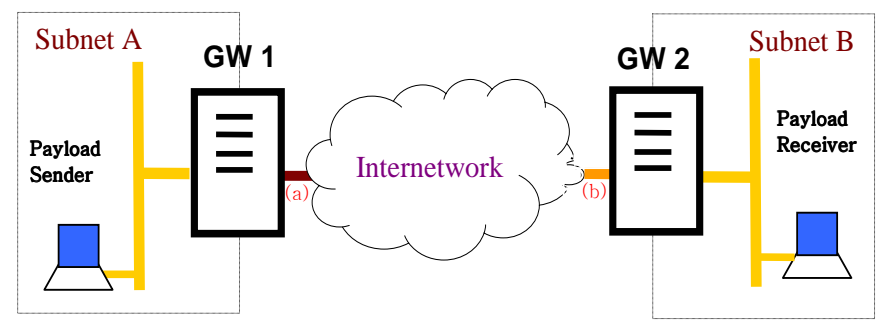

Figure 1. System Model

\subsection{Traffic Padding Mechanism}

The goal of the adversary is to perform traffic analysis and infer critical characteristics of the payload traffic exchanged over the unprotected network between protected subnets. We limit the interest of the adversary to the payload traffic rate, that is, the rate at which payload traffic is exchanged between protected networks. The traffic rate is a piece of important information in many mission critical communication applications [18]. Specifically, we assume that there is a set of discrete payload traffic rates $\left\{\omega_{1}, \cdots, \omega_{m}\right\}$. The rate of payload traffic from the sender may be one of those $m$ rates at a given time. Consequently, the objective of the adversary is to identify at which of the $m$ rates the payload is being sent.

One way to counter the traffic analysis attacks is to "pad" the payload traffic, that is, to properly insert "dummy" packets in the payload traffic stream so that the real payload status is camouflaged. There are many possible implementations of traffic padding algorithms on the two gateways in Figure 1. The most common method uses a timer to control packet sending, and works as follows:

- On GW1, incoming payload packets from the sender are placed in a queue.

- An interrupt-driven timer is set up on GW1. When the timer times out, the interrupt processing routine checks if there is a payload packet in the queue.

- If there are payload packets, one is removed from the queue and transmitted to GW2;

- Otherwise, a dummy packet is transmitted to GW2.

We need to make a few remarks before we proceed further.

- In this paper, we assume that packet contents are perfectly encrypted (e.g., by IPSec with appropriate options) and are thus non-observable. In particular, the adversary cannot distinguish between payload packets and "dummy" packets used for padding.

- It is obvious from the implementation described above, the only tunable parameter is the time interval between timer interrupts. The choice of this parameter discriminates different padding approaches.

- A system is said to have a constant interval timer (CIT) if the timer is a periodic one, i.e., the interval between two consecutive timer interrupts is constant. This is the most common method used for padding. 
- On the other hand, a system is said to have a variable interval timer (VIT) whenever the interval between two consecutive timer interrupts is a random variable and satisfies some distribution.

As we will see in the later part of this paper, CIT and VIT systems may perform significantly differently in preventing traffic analysis attacks.

- We assume that all packets have a constant size. Thus, observing the packet size will not provide any useful information to the adversary. The only information available for the adversary to observe and analyze is the timing of packets. This assumption should simplify the discussion without loss of the generality. See [10] for a discussion on how to extend our results in this paper to the case where packets may have variable sizes.

\subsection{Adversary Strategies}

Recall that we assume that the objective of the adversary is to identify at which of the $m$ possible rates the payload is being sent, and the adversary limits itself to passive attacks, i.e., observations of the traffic. In addition, the adversary's access to the system is limited to the unprotected networks. The protected subnets and the hosts within are not accessible. Neither is the link padding infrastructure. This means that, in Figure 1, the adversary can only tap somewhere between gateways GW1 and GW2.

We also assume that the adversary has complete knowledge about the gateway machines and the countermeasure algorithms used for preventing traffic analysis. For example, the adversary can simulate the whole system, including the gateway machines, to obtain a priori knowledge about traffic behavior. In many studies on information security, it is a convention that we make worst-case assumptions like this.

Based on these assumptions, the adversary can deploy a strategy based on Bayes decision theory [8]. The entire attack strategy consists of two parts: Off-line training and run-time classification. We now describe them below.

Off-line training The off-line training part can be decomposed into the following steps:

- The adversary selects a statistical feature of the Packet Inter-Arrival Time (PIAT) that will be used for traffic rate classification. Possible features we study in this paper are sample mean, sample variance, and sample entropy.

- The adversary reconstructs the entire traffic padding system and collects timing inforamtion at different payload traffic rates. From this information, the adversary derives the Probability Density Functions (PDF) of the selected statistical feature. As histograms are usually too coarse for the distribution estimation, we assume that the adversary uses the kernel estimator of PDF [20], which is effective in our problem domain. The kernel estimator of a density function with kernel $K$ is defined by

$$
f(s)=\frac{1}{M h} \sum_{i=1}^{M} K\left(\frac{s-S_{i}}{h}\right)
$$

where $h$ is the window width, also called the smoothing parameter or bandwidth, $S_{i}$ is the $i^{t h}$ observation of the feature statistic of padded traffic's PIATs, and $M$ is the number of such observations. In this paper, we use a Gaussian kernel, i.e., $K(s)=\frac{1}{\sqrt{2 \pi}} \exp \left(-\frac{s^{2}}{2}\right)$. 
- Based on the PDFs of statistical features for different payload traffic rates, Bayes decision rules are derived. Recall that there are $m$ possible payload traffic rates $\omega_{1}, \cdots, \omega_{m}$. The Bayes decision rule can be stated as follows:

The sample represented by feature s corresponds to payload rate $\omega_{i}$ if

$$
P\left(\omega_{i} \mid s\right) \geq P\left(\omega_{j} \mid s\right)
$$

That is,

$$
f\left(s \mid \omega_{i}\right) P\left(\omega_{i}\right) \geq f\left(s \mid \omega_{j}\right) P\left(\omega_{j}\right)
$$

for all $j=1, \cdots, m$.

Here $P\left(\omega_{i}\right)$ is the a priori probability that the payload traffic is sent at rate $\omega_{i}$, and $P\left(\omega_{i} \mid s\right)$ is the post priori probability that the payload traffic is sent at rate $\omega_{i}$ when the collected sample has the measured feature $s$.

Run-time Classifcation Once the adversary completes its training phase, he can start the classification at run time. We assume the adversary uses some means to tap the network between gateways GW1 and GW2. In particular, when he wants to determine the current payload rate, the adversary collects a sample of packet inter-arrival times. He calculates the value of the statistical feature from the collected sample, and then uses the Bayes decision rules derived in the training phase to match the collected sample to one of the previously defined payload traffic rates.

\section{Derivation of Detection Rate}

\subsection{Overview}

\subsubsection{Definition of Detection Rate}

Given the models described in the previous section, we would like to evaluate the system security in terms of detection rate. Detection rate is defined as the probability that the adversary can correctly classify the measured traffic, that is, identify the payload traffic rate. In this section, we derive the closed-form formulae for detection rates when the adversary uses sample mean, sample variance, or sample entropy, as the statistical feature, respectively. Our formulae will be approximate ones due to the complexity of the problem. Nevertheless, these formulae do correctly reflect the impact of various system parameters, including the type of padded traffic, the sample size, and the statistical feature used. These relationships are extremely useful in design of a traffic padding system so that the overall detection rate can be minimized. In the next section, we will see that experimental data well matchs the performance predicated by our approximation formulae.

We will focus our discussion on systems with only two payload traffic rates, namely $\omega_{l}$ as the low traffic rate and $\omega_{h}$ as the high traffic rate. We also assume that both traffic rates occur with equal probability. Extensions on this will be discussed in Section 6.

Figure 2 shows the PDFs of the statistical features conditioned on two alternative payload traffic rates. Let $d$ be the solution of the equation

$$
f\left(\omega_{l} \mid s\right)=f\left(\omega_{h} \mid s\right)
$$




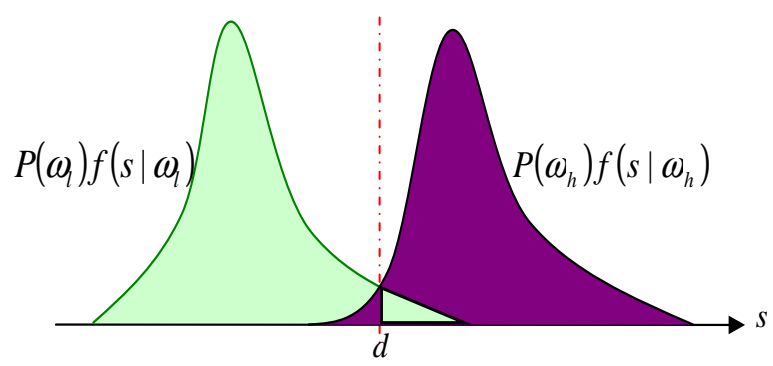

\section{Figure 2. Bayes Decision Making for the Case of Two Payload Traffic Rates}

and assume that there is a unique solution to the equation. Consequently, the Bayes decision rule now becomes

$$
\text { If } s \leq d \text {, the payload traffic rate is } \omega_{l} \text {; Otherwise, the rate is } \omega_{h} \text {. }
$$

The error rate for the Bayes decision rule can be calculated as follows:

$$
\epsilon=P\left(\omega_{h}\right) \int_{-\infty}^{d} f\left(s \mid \omega_{h}\right) d s+P\left(\omega_{l}\right) \int_{d}^{+\infty} f\left(s \mid \omega_{l}\right) d s
$$

The detection rate is then given by

$$
\begin{aligned}
v & =1-\epsilon \\
& =P\left(\omega_{l}\right) \int_{-\infty}^{d} f\left(s \mid \omega_{l}\right) d s+P\left(\omega_{h}\right) \int_{d}^{+\infty} f\left(s \mid \omega_{h}\right) d s
\end{aligned}
$$

While numerical methods can be applied to calculate the detection rates, for example with the use of (1), our goal here is to derive close-form formulae that can reveal the relationship between the detection rate and other system parameters.

\subsubsection{Decomposition of Packet Inter-Arrival Time}

Recall that the adversary collects a sample of packet inter-arrival times at run time in order to perform the classification. Thus, to derive the detection rate, we need to formally model the packet inter-arrival time. For a given system, let random variable $X$ be the packet inter-arrival time. $X$ can be considered as the sum of three other random variables:

$$
X=T+\delta_{g w}+\delta_{n e t}
$$

where $T$ is the designed interval of two consecutive timer interrupts for the timer, and $\delta_{g w}$ and $\delta_{n e t}$ reflect the noise added by disturbance in the gateway system and by congestion in the internetwork, respectively.

Note that $T$ is defined by the traffic padding policy. $T$ is constant for CIT traffic padding but follows a specific distribution for VIT traffic padding.

$\delta_{g w}$ may be caused by a number of factors, which may impact the accuracy of the timer's interrupt.

- First, the context switching from other running process to the timer's interrupt routine may take a random time. 
- Furthermore, a timer interrupt may be temporally blocked due to other activities. For example, if an payload packet from the sender is arriving at the network interface card of the gateway, the network interface card would generate an interrupt request, which can block all the processes including the (scheduled) timer interrupt ${ }^{1}$. Thus, the timer's interrupts may be subtly but randomly delayed by incoming payload packets. This implies that the padded traffic's PIAT may be correlated with the payload traffic.

$\delta_{n e t}$ captures the disturbance on the padded traffic's PIAT caused by congested routers and crossover traffic at routers and switches in the network. Clearly, $\delta_{n e t}$ depends on the position at which the adversary collects its sample. If the collection is done right at the output of the sender gateway, this noise may simply be ignored. However, if the adversary collects its sample far away from the sender gateway, then the noise level can be high as crossover traffic may significantly interfere with the padded traffic.

In this paper, we assume that both $T, \delta_{g w}$ and $\delta_{n e t}$ are normally distributed. These assumptions simplify analysis without loss of generality and will be validated by our experiments in Section 5. Specifically,

$$
T \sim N\left(\tau, \sigma_{T}^{2}\right)
$$

where $\sigma_{T}^{2}=0$ in the case of CIT traffic padding. And

$$
\delta_{\text {net }} \sim N\left(0, \sigma_{\text {net }}^{2}\right)
$$

where $\sigma_{n e t}^{2}=0$ when the adversary observes the padded traffic at a position next to the sender's gateway GW1. Similarly

$$
\delta_{g w} \sim N\left(0, \sigma_{g w}^{2}\right)
$$

As $\delta_{g w}$ may be correlated to the payload traffic, we denote $\sigma_{g w, l}^{2}$ and $\sigma_{g w, h}^{2}$ as the variances of $\delta_{g w}$ when the payload traffic rate is low and high, respectively. Consequently, we denote $X_{l}$ and $X_{h}$ are random variable $X$ when the payload traffic rate is low and high, respectively. Thus,

$$
X_{l} \sim N\left(\mu, \sigma_{l}^{2}\right)
$$

where $\mu=\tau$ and

$$
\sigma_{l}^{2}=\sigma_{T}^{2}+\sigma_{n e t}^{2}+\sigma_{g w, l}^{2}
$$

Similarly,

$$
X_{h} \sim N\left(\mu, \sigma_{h}^{2}\right)
$$

where $\mu=\tau$ and

$$
\sigma_{h}^{2}=\sigma_{T}^{2}+\sigma_{n e t}^{2}+\sigma_{g w, h}^{2}
$$

\footnotetext{
${ }^{1}$ For TimeSys Linux [25] used in our experiments, this request proceeds before the incoming packet reaches the IP layer [11]. From that instant on, the network subsystem in the kernel becomes preemptive. Other high priority tasks such as the timer interrupt routine can then proceed as scheduled.
} 
Here we assume that $X_{l}$ and $X_{h}$ have the same mean. This assumption will be validated by our experiments later.

For the convenience of the discussion in the rest of this paper, we need to introduce the ratio

$$
r=\frac{\sigma_{h}^{2}}{\sigma_{l}^{2}}=\frac{\sigma_{T}^{2}+\sigma_{n e t}^{2}+\sigma_{g w, h}^{2}}{\sigma_{T}^{2}+\sigma_{n e t}^{2}+\sigma_{g w, l}^{2}}
$$

where $\sigma_{T}^{2}, \sigma_{n e t}^{2}, \sigma_{g w, l}^{2}$ and $\sigma_{g w, h}^{2}$ are defined in (10), (11), (14), and (16), respectively. The use of $r$ will become clear when we derive the formulae for detection rates for three different statistical features, namely, sample mean, sample variance, and sample entropy.

\subsection{The Case of Sample Mean}

\subsubsection{Basic Result}

Let $\left\{X_{1}, X_{2}, \cdots, X_{n}\right\}$ be a random sample of packet inter-arrival times. The sample mean is the average of the elements in the sample:

$$
\bar{X}=\frac{\sum_{i=1}^{n} X_{i}}{n}
$$

Note that sample mean $\bar{X}$ is a random variable, and an unbiased estimation of $X$ 's mean $\mu$.

The following theorem provides a closed-form formula for estimation of detection rate when the adversary uses sample mean as the feature statistic.

Theorem 1 When sample mean is used as the feature statistic, the detection rate can be estimated as follows

$$
v_{\bar{X}} \approx 1-\frac{1}{\sqrt{2(1 / \sqrt{r}+\sqrt{r})}}
$$

where $r$ is defined in (17).

From Theorem 1 the following observations can be made:

1. The detection rate in (19) is independent on sample size $n$. That is, when sample mean is used as feature statistic, changing the sample size has no impact on detection rates.

2. As shown in Appendix A, the detection rate $v_{\bar{X}}$ is an increasing function of $r$, where $r \geq 1$. That is, the smaller $r$, the lower the corresponding detection rate. When $r=1$, the detection rate reaches $50 \%$ - its absolute lower bound. In reality, $r=1$ may occur when $\sigma_{T}^{2}$ is sufficiently large. This corresponds to the case when the VIT padding is used.

\subsubsection{Proof of Theorem 1}

Proof: Theorem 1 can be proved as follows. The distribution of sample mean for a normal distribution $N\left(\mu, \sigma^{2}\right)$ is still a normal one, $N\left(\mu, \frac{\sigma^{2}}{n}\right)$. Thus, sample mean $\bar{X}_{l}$ for the case when the payload traffic rate is low has a normal distribution

$$
f_{l}(x)=N\left(\mu, \sigma_{1}^{2}\right)=N\left(\mu, \frac{\sigma_{l}^{2}}{n}\right) .
$$


Similarly, sample mean $\bar{X}_{h}$ for the high payload traffic rate has a normal distribution

$$
f_{h}(x)=N\left(\mu, \sigma_{2}^{2}\right)=N\left(\mu, \frac{\sigma_{h}^{2}}{n}\right)
$$

Since $\bar{X}_{l}$ and $\bar{X}_{h}$ are normally distributed, we can use the Bhattacharyya bound [8] to estimate the error rate as follows:

$$
\epsilon_{\bar{X}} \leq \sqrt{P\left(\omega_{l}\right) P\left(\omega_{h}\right)} \int \sqrt{f\left(x \mid \omega_{l}\right) f\left(x \mid \omega_{h}\right)} d x
$$

Substituting (20), (21), and $P\left(\omega_{l}\right)=P\left(\omega_{h}\right)=0.5$ into (22) and carrying out the integration, we have

$$
\epsilon_{\bar{X}} \leq \frac{1}{2} e^{-k}
$$

where

$$
k=\frac{1}{2} \ln \frac{\frac{\sigma_{l}^{2}+\sigma_{h}^{2}}{2}}{\sqrt{\sigma_{l}^{2} \sigma_{h}^{2}}}
$$

After substituting (17) into (24) and some rearranging, we have

$$
k=\frac{1}{2} \ln \frac{(1 / \sqrt{r}+\sqrt{r})}{2}
$$

Substituting (25) into (23), the error rate is given by

$$
\epsilon_{\bar{X}} \leq \frac{1}{\sqrt{2(1 / \sqrt{r}+\sqrt{r})}}
$$

The detection rate $v_{\bar{X}}$ then satisfies the following:

$$
\begin{aligned}
v_{\bar{X}} & =1-\epsilon_{\bar{X}} \\
& \geq 1-\frac{1}{\sqrt{2(1 / \sqrt{r}+\sqrt{r})}}
\end{aligned}
$$

Thus, we can use the lower bound of (28) as the estimation of detection rate by sample mean. The theorem is proven.

\subsection{The Case of Sample Variance}

Let $\left\{X_{1}, X_{2}, \cdots, X_{n}\right\}$ be a random sample of size $n$ from the distribution of $X$. The sample variance $Y$ is defined as follows

$$
Y=\frac{\sum_{i=1}^{n}\left(X_{i}-\bar{X}\right)^{2}}{n-1}
$$

Note that sample variance $Y$ is a random variable, and an unbiased estimation of $X$ 's variance.

Recall that $\sigma_{h}^{2}$ is the variance of padded traffic's PIAT conditioned on the high payload traffic rate and $\sigma_{l}^{2}$ the variance of padded traffic's PIAT conditioned on the low payload traffic rate. $\sigma_{h}^{2}$ is slightly larger than $\sigma_{l}^{2}$, which is validated by our experiments in Section 5. Based on these observations, the following theorem provides a closed-form formula for estimation of detection rate when the adversary uses sample variance as the feature statistic. 
Theorem 2 Using sample variance with sample size $n$ as the classification feature gives rise to an estimated detection rate $v_{Y}$

$$
v_{Y} \approx \max \left(1-\frac{C_{Y}}{n-1}, 0.5\right)
$$

where $C$ is calculated in (31).

$$
C_{Y}=\frac{1}{2\left(1-\frac{1}{r-1} \log r\right)^{2}}+\frac{1}{2\left(\frac{r}{r-1} \log r-1\right)^{2}}
$$

and $r=\frac{\sigma_{h}^{2}}{\sigma_{l}^{2}}$ as defined in (17).

The proof of Theorem 2 is lengthy. It can be found in the first part of Appendix B. From Theorem 2 the following observations can be made:

1. The detection rate $v_{Y}$ is an increasing function in terms of sample size $n$. When $n \rightarrow \infty$, the detection rate is $100 \%$. This means that the payload traffic lasts for a long time at one rate, either low or high, the adversary gets such a sample and may detect the payload traffic rate by sample variance of padded traffic's PIAT.

2. As shown in the second part of Appendix $\mathrm{B}$, the detection rate $v_{Y}$ is an increasing function of $r$ in (17), where $r \geq 1$. That is, the smaller $r$, the lower the corresponding detection rate. When $r=1$, the detection rate is $50 \%$. This corresponds to the case when VIT padding with sufficiently large $\sigma_{T}^{2}$. This suggests that although the adversary may use a big size of sample to detect the payload rate by sample variance, using a VIT padding with a large interval variance can make such an attack impossible, since no payload traffic can last very long at a fixed rate in practice and the adversary cannot get a sample big enough.

\subsection{The Case of Sample Entropy}

While there are many empirical entropy estimators available, it's generally very difficult to get those estimators' PDFs. In this work, we take advantage of the relation between entropy and variance of a normal distribution in order to describe sample entropy's effectiveness as the feature statistic. We will then use an empirical robust histogram-based entropy estimator for our experiments.

The following theorem provides a closed-form formula for estimation of detection rate when the adversary uses sample entropy as the feature statistic.

Theorem 3 Sample entropy with sample size $n$ has an estimated detection rate $v_{\tilde{H}}$

$$
v_{\tilde{H}} \approx \max \left(1-\frac{C_{H}}{n}, 0.5\right)
$$

where $C_{\tilde{H}}$ is calculated in (33)

$$
C_{\tilde{H}}=\frac{1}{2\left(\log \left(\frac{r}{r-1} \log r\right)\right)^{2}}+\frac{1}{2\left(\log \left(\frac{r-1}{\log r}\right)\right)^{2}}
$$

and $r=\frac{\sigma_{h}^{2}}{\sigma_{l}^{2}}$ as defined in (17). 
The proof of Theorem 3 can be found in the first part of Appendix C. From Theorem 3 we can make a similar set of observations to that of the case of sample variance.

1. Detection rate $v_{\tilde{H}}$ is an increasing function in terms of sample size $n$.

2. As shown in the second part of Appendix C, the detection rate $v_{\tilde{H}}$ is an increasing function of $r$ in (17), where $r \geq 1$. When $r=1$, the detection rate reaches $50 \%$. In reality, $r=1$ may occur when $\sigma_{T}^{2}$ is sufficiently large. This corresponds to the case when VIT padding with sufficiently large $\sigma_{T}^{2}$ is used.

From statistical knowledge, we know sample variance is very sensitive to outliers ${ }^{2}$. In order for empirical estimation of sample entropy to be robust against outliers, we use the method developed in [14]: First, we create a histogram of the PIAT sample for a given bin size (say, $\Delta h$ ). Then, according to [14], the differential entropy estimator of a random variable $X$ 's continuous distribution is

$$
\widetilde{H} \approx-\sum_{i} \frac{k_{i}}{n} \log \frac{k_{i}}{n}+\log \Delta h
$$

where $n$ is the sample size, $k_{i}$ is the number of sample points in the $i^{t h}$ bin, and $\Delta h$ is the histogram's bin size. If a constant bin size is used throughout the experiment, the term $\log \Delta h$ in (34) is a constant and hence does not influence the recognition result. It can therefore be discarded, and the entropy estimation formula simplifies to

$$
\widetilde{H} \approx-\sum_{i} \frac{k_{i}}{n} \log \frac{k_{i}}{n}
$$

This entropy estimator is robust in the sense that it is based on probability weighted sum. Generally, outliers have a small probability to occur. So the probability weight reduces the noise's impact on the entropy estimation. Moreover, from the discussion in [14] and our experiments, we found that this histogram-based entropy estimator matches Theorem 3.

\section{Evaluations}

In this section, we report results on evaluating system security in terms of detection rate. The evaluations will be based on both theoretical analysis (from the previous section) and real experiments.

In the experiments, we assume that the adversary uses a high-performance network analyzer, such as Agilent's J6841A [24], to dump the padded traffic for traffic analysis. A series of experiments were carried out: In terms of padded traffic type, we measure both systems with CIT and VIT padding, In terms of experimental environments, we consider the following cases: a) a laboratory environment, b) a campus network, and c) a wide area network.

In all of our experiments, GW1 and GW2 in Figure 1 are implemented using TimeSys Linux/Real-Time [25]. Both CIT and VIT paddings are based on timer interrupts with interval mean equal to $10 \mathrm{~ms}$, i.e., $E(T)=10 \mathrm{~ms}$ where $T$ is defined in (9). We consider that the payload has two rate states: 10 packet per second (pps) and 40pps. We assume both rates occur in equal probability, that is, $P\left(\omega_{l}\right)=P\left(\omega_{h}\right)=50 \%$ in (8). Note that for such a system with two possible payload traffic rates, the detection rate for the adversary is lower-bounded at 50\%, as a random guess would result in a detection rate of $50 \%$.

\footnotetext{
${ }^{2}$ An outlier is an observation that lies an abnormal distance from other values in the sample of the padded traffic PIAT.
} 


\subsection{Experiments in a Laboratory Environment}

The advantage of performing the experiments in a laboratory environment is that we can control the cross traffic over the network. The disadvantage is that the generated cross traffic may not have the same characteristics as that in a real network. Nevertheless, our experiment setup is shown in Figure 3.

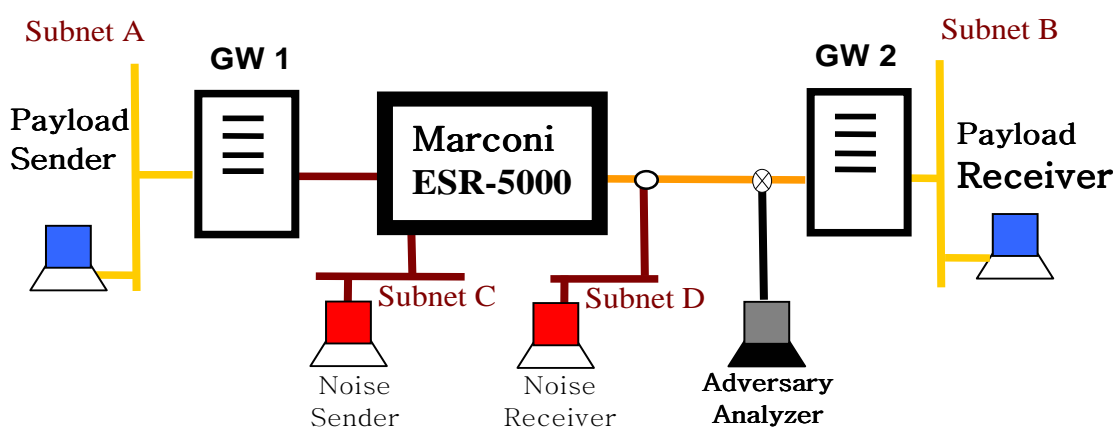

Figure 3. Experiment setup in laboratory

The two gateways are connected by a Marconi ESR-5000 enterprise switching router [17]. Subnet C is connected to the Marconi router as the cross traffic (noise) generator while the cross traffic receiver is located in Subnet D. Note that the cross traffic shares the outgoing link of the router, creating a case that the cross traffic makes an impact over the padded traffic.

\subsubsection{The Case of Zero Cross Traffic}

Here, we consider the case when there is no cross traffic. That is, the workstation in subnet $\mathrm{C}$ does not transmit, and the router only deals with the padded traffic from GW1. That is, $\sigma_{\text {net }}$ in (17) is 0 . Hence, the variance ratio $r$ becomes

$$
r=\frac{\sigma_{T}^{2}+\sigma_{g w, h}^{2}}{\sigma_{T}^{2}+\sigma_{g w, l}^{2}}
$$

This situation is a best case for the adversary as it can observe traffic with minimum disturbance. Hence this is the worst-case for us who wants to prevent traffic analysis attacks.

\section{CIT Traffic Padding}

First, we analyze systems that use CIT traffic padding. That is, $\sigma_{T}^{2}$ in (17) is zero. Hence, (36) is further simplified as

$$
r=\frac{\sigma_{g w, h}^{2}}{\sigma_{g w, l}^{2}}
$$

From the theorems in Section 4, we see that the detection rates are functions of $n$, sample size and $r$, the ratio of the variance of padded traffic's PIAT in two different rate states.

Figure 4 (a) shows the distributions of padded traffic's PIAT under low-rate (10pps) and high-rate (40pps) payload traffic. We have the following observations:

1. The two distributions are almost bell-shaped. This partially validates our assumption that the padded traffic's PIAT has a normal distribution. 

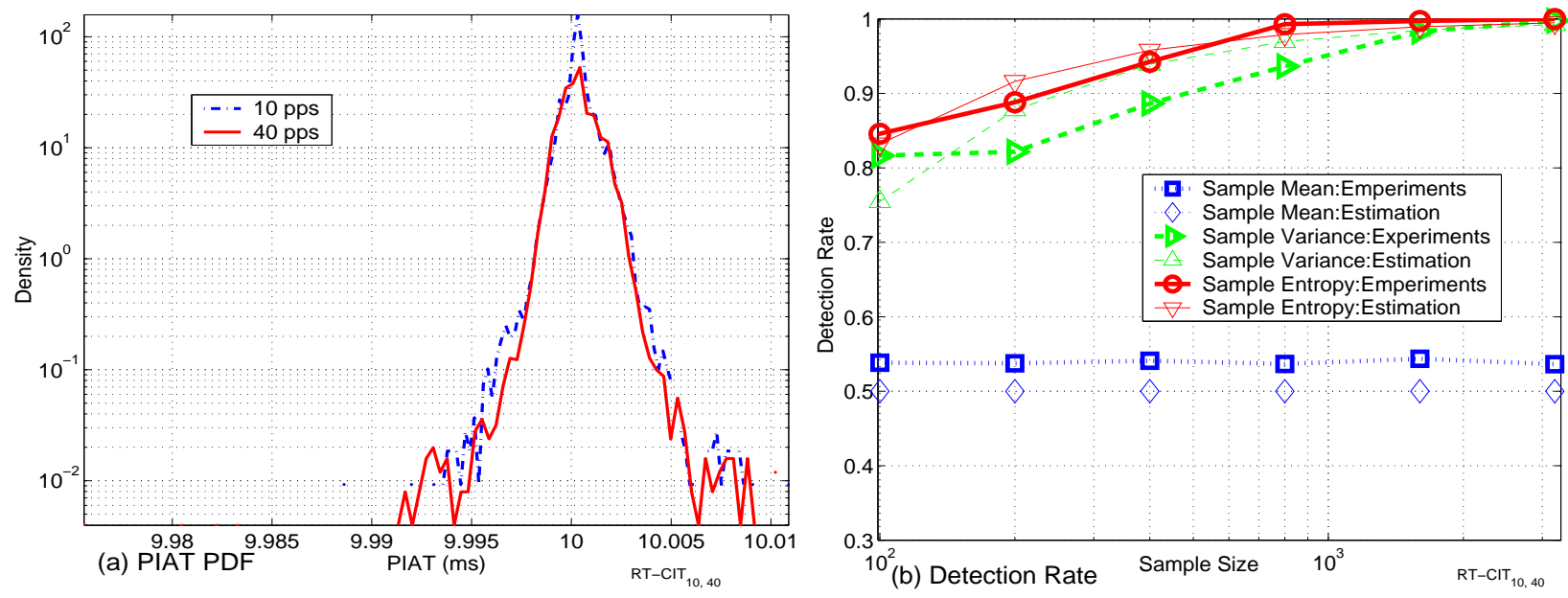

Figure 4. CIT Padding without cross traffic

2. The means of padded traffic's PIAT under different rates of payload traffic are the same. This is also consistent with the assumption made in Section 4.2.

3. The two distributions are slightly different and the variance of padded traffic's PIAT conditioned on the high-rate payload traffic, $\sigma_{g w, h}^{2}$ in (16), is slightly larger than the variance of padded traffic's PIAT conditioned on the low-rate payload traffic, $\sigma_{g w, l}^{2}$ in (14). This implies

$$
r=\frac{\sigma_{g w, h}^{2}}{\sigma_{g w, l}^{2}}>1
$$

Figure 4 (b) shows both empirical and theoretical curves of detection rate for different feature statistics. From this data, we make the following observations:

1. The empirical detection rate curves coincide well with their theoretical curves. This validates our theories developed in Section 4. The empirical detection rate curve of sample variance is a little lower than its theoretical curve. This is because sample variance is very sensitive to outliers and sometimes it is difficult to correctly identify outliers [7].

2. The detection rate of sample mean is almost $50 \%$. CIT is an effective padded traffic type to prevent traffic analysis attacks if the adversary uses sample mean as the feature statistic.

3. On the other hand, as the sample size increases, detection rates for both sample variance and sample entropy increase as predicated by our theorems 1 and 3. As the sample size reaches 1,000, both of these statistics achieve almost $100 \%$ detection rate. This means that CIT is not an effective padding approach to prevent traffic analysis attacks if the adversary uses sample variance or sample entropy as feature statistic. Generally speaking, sample entropy performs empirically better than sample variance in terms of detection rate.

\section{VIT Traffic Padding}


Recall from (36) how the variance ratio $r$ in (17) is given by

$$
r=\frac{\sigma_{T}^{2}+\sigma_{g w, h}^{2}}{\sigma_{T}^{2}+\sigma_{g w, l}^{2}}
$$

where $\sigma_{T}^{2} \geq 0$ since we are using VIT padding.

From our theorems in Section 4, we know that when $r$ approaches 1, the detection rates approach 50\% for all the three feature statistics. We note that for CIT padding, the value of $r$ decreases with increasing values of $\sigma_{T}^{2}$. Figure 5 (a) displays the empirical curves of detection rate in terms of $\sigma_{T}$ for a fixed sample size of 2,000. We can see that when $\sigma_{T}$ increases, the detection rate quickly drops and approaches $50 \%$, as expected. Clearly, a system with VIT padding performs better (i.e., with lower detection rate) than one with CIT padding.

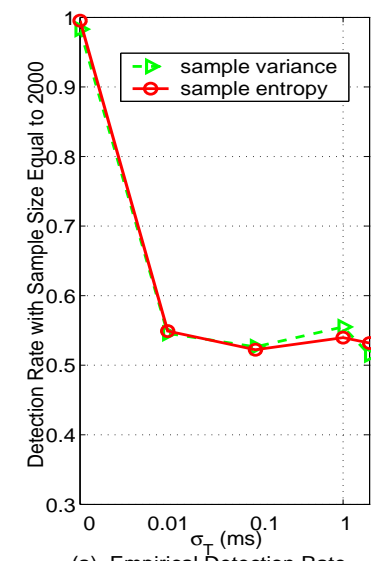

(a) Empirical Detection Rate

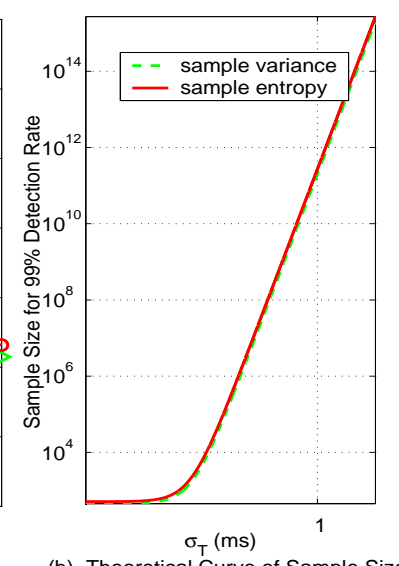

(b) Theoretical Curve of Sample Size

Figure 5. VIT padding - detection rate vs. sample size

In any case, as shown in (19) and (32), when the size of sample increases, the detection rate increases as well. An interesting question is: How large a sample has to be in order for the adversary to have sufficient high probability in making a correct detection? Let $n(p)$ be the sample size that can achieve a detection rate of $p$ percent. Figure 5 (b) provides the theoretical curve of $n(99 \%)$ vs. $\sigma_{T}$. We can see that with a reasonable value of $\sigma_{T}$, the sample size needs to be extremely large in order to achieve a 99\% detection rate. For example, when the timer interval standard deviation $\sigma_{T}=1 \mathrm{~ms}$, to achieve $99 \%$ detection rate, the sample size has to be greater than $10^{11}$. It is virtually impossible for an attacker to retrieve such a large sample. This clearly shows the effectiveness of VIT padding.

\subsection{The Case of Non-Zero Cross Traffic}

Recall that the case of zero cross traffic is the best case for the adversary. As VIT has shown to be effective in the case of zero cross traffic, we will no longer have to consider systems with VIT padding here since VIT has been shown to be effective even for the adversary's best-case scenario (zero cross-traffic with a line tap very near the sender gateway). We thus concentrate on the system with CIT padding. In a system with cross traffic, $\sigma_{n e t}^{2}$ in (17) may no longer be zero. As for CIT 
padding, where $\sigma_{T}^{2}=0$, the variance ratio $r$ in (17) now becomes

$$
r=\frac{\sigma_{n e t}^{2}+\sigma_{g w, h}^{2}}{\sigma_{n e t}^{2}+\sigma_{g w, l}^{2}}
$$

We observe that $r$ decreases with increasing $\sigma_{n e t}^{2}$, resulting in a low detection rate for all feature statistics. Thus, the bigger $\sigma_{n e t}^{2}$, the smaller the detection rate.

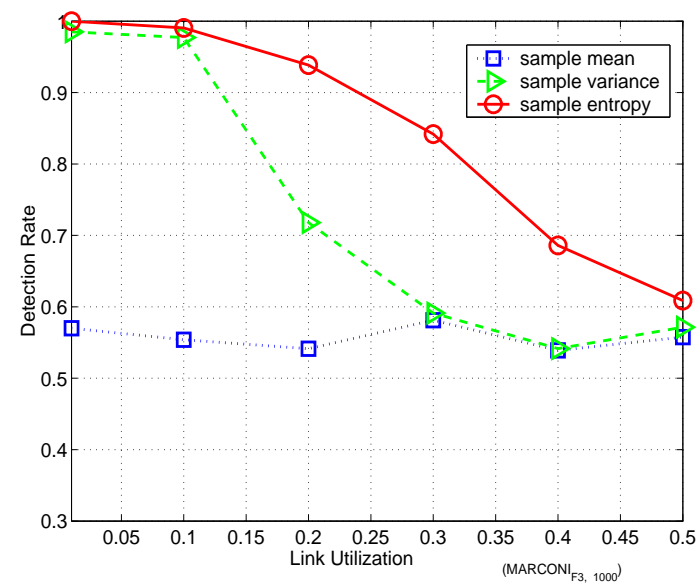

Figure 6. Empirical detection rate with cross traffic in laboratory

In the experiments described here, cross traffic generated from in subnet $\mathrm{C}$ causes the router's congestion, which in turn affects the obervation by the adversary. Figure 6 shows how the detection rate is impacted by the amount of cross traffic. We can make the following observations:

1. Note that the PIAT for the padded traffic is $10 \mathrm{~ms}$. Hence, the amount of cross traffic is directly proportional to the utilization of the link shared between Subnet B and Subnet D. The data shows that as the link utilization increases, the detection rate by sample entropy and sample variance decrease. Intuitively, this is because the crossover traffic between Subnet C and Subnet D interferes with the padded traffic between GW1 and GW2, and $\sigma_{n e t}^{2}$ increases with the shared link's utilization. The sample mean's detection rate remains low, as expected.

2. We observe that sample entropy results in a better detection rate than sample variance does. It can be perceived that, with the increase of shared link's utilization, outliers have more chance of occurring. Sample variance is much more sensitive to outliers and, hence, it has a low detection rate.

3. Even with the link utilization of $40 \%$, sample entropy still can have about a detection rate of $70 \%$, implying that CIT padding may still not be effective in this kind of situation.

\subsection{Experiments over Campus and Wide Area Networks}

Figure 7 shows the setup for the experiments discussed in this subsections. Figure 7 (a) is a setup for experiments over the Texas A\&M Campus Network. That is, the padded traffic goes through Texas A\&M campus network before it reaches the 


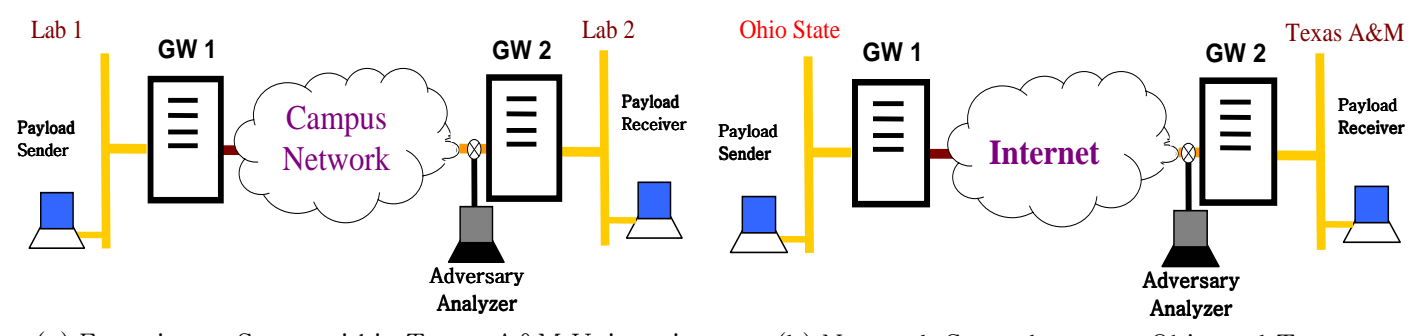

(a) Experiment Setup within Texas A\&M University

(b) Network Setup between Ohio and Texas

Figure 7. Experiment setup over campus and wide area networks (WAN)

receiver's gateway. Figure 7 (b) is a setup for experiments over the Internet between Ohio State University and Texas A\&M University. Here, the sender workstation and the sender gateway are located at Ohio State University. The padded traffic goes through the Internet and arrives at Texas A\&M University, where the receiver gateway and the receiver's workstation are located. In both cases, the observation point of the adversary is located right in front of the receiver gateway and thus maximally far from the sender. We note that in this case, the path from the sender's workstation to the receiver's workstation spans over 15 routers.

In each case, we collect data continuously for a complete day (24 hours). The data for the case of Texas A\&M campus network was collected on March 24, 2003 while the data for the wide are network case was collected on March 26, 2003.
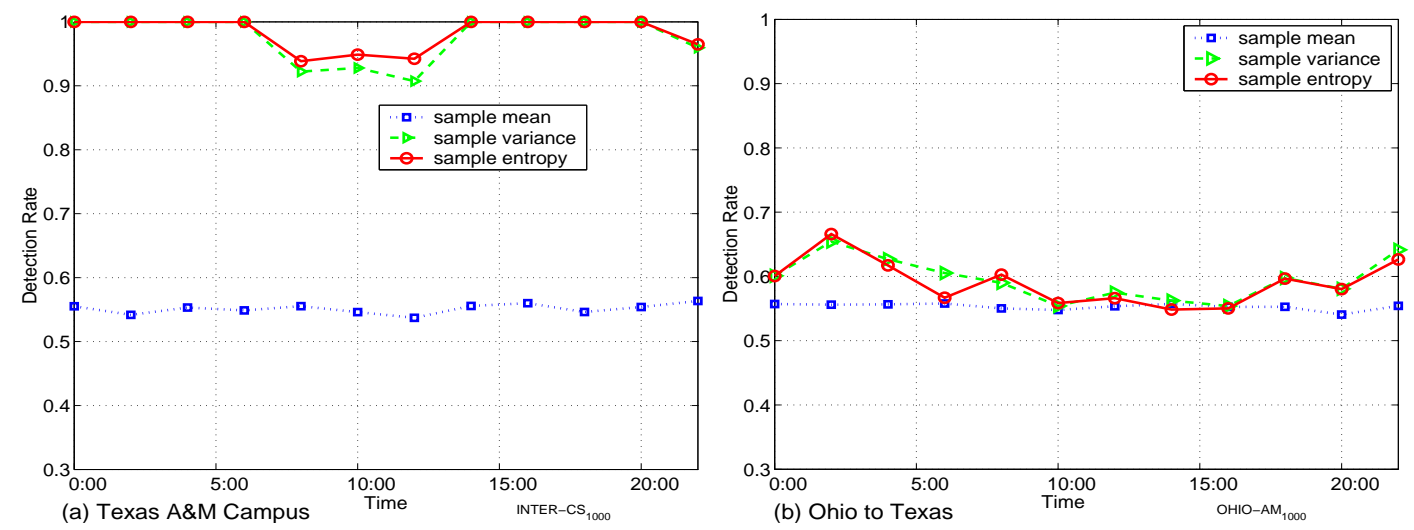

Figure 8. Empirical detection rates for experiments over campus and WAN (sample size=1000)

Figures 8 (a) and (b) display the detection rate throughout the observation period. We make the following observations:

1. When the padded traffic traverses just the Texas A\&M campus network, the detection rates of sample entropy and sample variance are high almost all the time period in the day we collected data. This means that over a medium-size enterprise network like the Texas A\&M campus, the crossover traffic has limited influence on the padded traffic's PIAT. Consequently, we would not recommend CIT padding to be used in such an environment.

2. When the padded traffic traverses more network elements, such as the span of the Internet between Ohio State Univer- 
sity and Texas A\&M University, the detection rates are lower. This is because the padded traffic experiences congestion at a large number of routers and switches, and its PIAT is seriously distorted with a relatively large $\sigma_{n e t}^{2}$.

3. In the case of wide area networks, sample entropy and sample variance can still get over $65 \%$ detection rates during periods of relatively low network activity (such as at 2:00AM). This means that CIT padding may still not be sufficiently safe even if the adversary is very remote.

\section{Conclusions and Final Remarks}

While researchers have proposed traffic padding as effective ways to prevent traffic analysis, before this study there has been no systematic method to analyze the information assurance of a security system under the attack of traffic analysis. This paper gives an effective analysis model for the evaluation of different padding strategies aimed at camouflaging the payload traffic rates under the attack of traffic analysis. We define as our security metric detection rate, which is the probability that the payload traffic is recognized. We believe that our analysis methods can be widely used to analyze other security systems for different objectives under traffic analysis attacks.

By statistical analysis of different feature statistics (sample mean, sample variance and sample entropy) of the padded traffic's packet interarrival times and a lot of experiments, we found that sample variance and sample entropy can exploit the correlation between payload traffic rate and packet interarrival times of padded traffic when the padded traffic is dumped and explored next to the sender gateway or at a remote site across one or more congested routers. The reason for CIT padding's failure is that user traffic causes small disturbances to the timer's interval, which is used to control packet sending. Moreover, the higher the user traffic rate, the larger the disturbance of the padded traffic's PIAT.

After a careful analysis, we propose VIT traffic padding as an alternative to the most common CIT traffic padding. Both theoretical analysis and empirical results validate the effectiveness of VIT padding strategy. The importance of VIT padding technique is validated by extensive experiments showing that CIT link padding may be compromised even at a remote site behind noisy routers.

In this paper we discuss the simple case where two classes of traffic rates should be distinguished. Our technique can be easily extended to multiple ones, in which case more off-line training is needed. We also assume that the payload traffic from the user has a constant packet size. Recent measurements $([3,6])$ indeed indicate that the size of packets on the Internet can be described using distributions, with most of the packets distributed at a few fixed sizes. According to this a priori knowledge (maybe in addition to specific knowledge particular to the environment), the user traffic fitting these distributions can be simulated. This allows the design of link padding schemes that are tuned to this a priori knowledge to prevent traffic analysis.

\section{References}

[1] Onion Routing Development Achives. Link padding and the intersection attack. http://archives.seul.org/or/dev/Aug2002/msg00004.html, 82002. 
[2] P. Baran. On distributed communications: Ix security, secrecy, and tamper-free considerations. Memo RM-3765-PR, Rand Corp., Aug. 1964.

[3] CAIDA. Packet sizes and sequencing. http://www.caida.org/outreach/resources/learn/packetsizes/, June 2002.

[4] George Casella and Roger L. Berger. Statistical inference. Australia ; Pacific Grove, CA : Duxbury/Thomson Learning, second edition, 2002.

[5] D. L. Chaum. Untraceable electronic mail, return addresses, and digital pseudonyms. Communications of the ACM, 24(2), Feb. 1981.

[6] K. claffy, G. Miller, and K. Thompson. the nature of the beast: recent traffic measurements from an internet backbone. Proceedings of ISOC INET' '98, July 1998.

[7] Ralph B. D’Agostino and Michael A. Stephens. Goodness-of-fit techniques. Marcel Dekker, inc., 1986.

[8] R. O. Duda and P. E. Hart. Pattern Classification. John Wiley \& Sons, 2001.

[9] Edward W. Felten and Michael A. Schneider. Timing attacks on web privacy. ACM Conference on Computer and Communications Security (CCS), 2000.

[10] Xinwen Fu. Traffic Analysis Attacks and Countermeasures. PhD thesis, Texas A\&M University, College Station, TX, USA, 2003.

[11] S. Ghosh and R. Rajkumar. Resource management of the os network subsystem. Proceedings of the Fifth IEEE International Symposium on Object-Oriented Real-Time Distribute Computing, April 2002.

[12] Y. Guan, X. Fu, D. Xuan, P. U. Shenoy, R. Bettati, and W. Zhao. Netcamo: Camouflaging network traffic for qosguaranteed critical allplications. In IEEE Transactions on Systems, Man, and Cybernetics Part A: Systems and Humans, Special Issue on Information Assurance, volume 31 of 4, pages 253-265, July 2001.

[13] SafeWeb inc. Safeweb. http://www.safewebinc.com/, 2002.

[14] R. Moddemeijer. On estimation of entropy and mutual information of continuous distributions. Signal Processing, 16(3):233-246, 1989.

[15] R. E. Newman-Wolfe and B. R. Venkatraman. High level prevention of traffic analysis. Computer Security Applications Conference, Seventh Annual, pages 102 -109, 1991.

[16] R. E. Newman-Wolfe and B. R. Venkatraman. Performance analysis of a method for high level prevention of traffic analysis. Computer Security Applications Conference, Eighth Annual, pages 123 -130, 1992.

[17] Marconi Corporation plc. Esr-5000 and esr-6000 enterprise switch routers. http://www.marconi.com/html/products/esr50006000.htm, 2003. 
[18] J. Raymond. Traffic analysis: Protocols, attacks, design issues and open problems. In H. Federrath, editor, Designing Privacy Enhancing Technologies: Proceedings of International Workshop on Design Issues in Anonymity and Unobservability, volume 2009 of LNCS, pages 10-29. Springer-Verlag, 2001.

[19] C. E. Shannon. Communication theory of secrecy systems. Bell Sys. Tech. J., 28:656-715, 1949.

[20] B. W. Silverman. Density estimation for statistics and data analysis. Chapman and Hall, London, New York, 1986.

[21] D. X. Song, D. Wagner, and X. Tian. Timing analysis of keystrokes and timing attacks on ssh. 10th USENIX Security Symposium, 2001.

[22] Qixiang Sun, Daniel R. Simon, Yi-Min Wang, Wilf Russell, Venkata N. Padmanabhan, and Lili Qiu. Statistical identification of encrypted web browsing traffic. IEEE Symposium on Security and Privacy, May 2002.

[23] P. F. Syverson, D. M. Goldschlag, and M. G. Reed. Anonymous connections and onion routing. In IEEE Symposium on Security and Privacy, pages 44-54, Oakland, California, 4-7 1997.

[24] Agilent Technologies. Agilent j6841a network analyzer software. http://onenetworks.comms.agilent.com/NetworkAnalyzer /J6841A.asp, March 2002.

[25] TimeSys. Timesys linux docs. http://www.timesys.com/index.cfm? hdr=home_header.cfm\&bdy=home_bdy_library.cfm, 2003.

[26] Brenda Timmerman. a security model for dynamic adaptive traffic masking. New Security Paradigms Workshop, 1997.

[27] B. R. Venkatraman and R. E. Newman-Wolfe. Performance analysis of a method for high level prevention of traffic analysis using measurements from a campus network. Computer Security Applications Conference, 10th Annual, pages $288-297,1994$.

[28] V. Voydoc and S. Kent. Security mechanisms in high-level network protocols. ACM Computing Surveys, pages 135 $171,1983$.

\section{Appendix A}

In this appendix, we prove that the detection rate $v_{\bar{X}}$ by sample mean is an increasing function of $r$.

To prove $v_{\bar{X}}$ increases with $r$, we need prove that the term $t(r)=\sqrt{2(1 / \sqrt{r}+\sqrt{r})}$ in (19) increases with $r$.

$$
\left.d t / d r=\frac{1}{2 \sqrt{r}}\left(1-\frac{1}{\sqrt{r}}\right)\right)
$$

Since $r \geq 1, d t / d r \geq 0$. Thus $t(r)$ increases with $r$, and the detection rate $v_{\bar{X}}$ by sample mean increases with $r$ too. 


\section{Appendix B}

(1) In this part, we prove Theorem 2.

Proof: Denote $\chi_{n-1}^{2}$ as a random variable with a chi square distribution $f_{\chi_{n-1}^{2}}(x)$ with freedom $n-1$, which is defined as follows,

$$
f_{\chi_{n-1}^{2}}(x)=\frac{x^{\frac{n-1}{2}-1} e^{-\frac{x}{2}}}{2^{\frac{n-1}{2}} \Gamma\left(\frac{n-1}{2}\right)}
$$

where $x>0$. Denote $Y$ as the random variable of sample variance. Then $\frac{n-1}{\sigma^{2}} Y$ has a chi square distribution with freedom $n-1$ [4]. That is

$$
\chi_{n-1}^{2}=\frac{n-1}{\sigma^{2}} Y
$$

From (42), we get

$$
Y=\frac{\sigma^{2}}{n-1} \chi_{n-1}^{2}
$$

From chi square's properties, we get sample variance's mean $\bar{Y}$ as

$$
\bar{Y}=\sigma^{2}
$$

and its variance $\operatorname{var}(Y)$ as

$$
\operatorname{var}(Y)=\frac{2 \sigma^{4}}{n-1}
$$

To get sample variance's PDF at sample size $n$, we first compute its distribution function

$$
P(Y<y)=P\left(\frac{\sigma^{2}}{n-1} \chi_{n-1}^{2}<y\right)=P\left(\chi_{n-1}^{2}<\frac{n-1}{\sigma^{2}} y\right)
$$

Differentiating the two sides of (46), we have the density function

$$
f_{Y}(y)=f_{\chi_{n-1}^{2}}\left(\frac{n-1}{\sigma^{2}} y\right) \frac{n-1}{\sigma^{2}}
$$

We denote $Y_{l}$ as the random variable of sample variance of padded traffic's PIAT at low rate payload traffic, substitute (41) into (47), and then derive $Y_{l}$ 's density function $f_{Y_{l}}(y)$

$$
f_{Y_{l}}(y)=\frac{\left(\frac{n-1}{\sigma_{l}^{2}} y\right)^{\frac{n-1}{2}-1} e^{-\left(\frac{n-1}{2 \sigma_{l}^{2}} y\right)}}{2^{\frac{n-1}{2}} \Gamma\left(\frac{n-1}{2}\right)} \frac{n-1}{\sigma_{l}^{2}}
$$

Similarly, $Y_{h}$ is the random variable of sample variance of padded traffic's PIAT at high rate payload traffic, and its density function $f_{Y_{h}}(y)$ is

$$
f_{Y_{h}}(y)=\frac{\left(\frac{n-1}{\sigma_{h}^{2}} y\right)^{\frac{n-1}{2}-1} e^{-\left(\frac{n-1}{2 \sigma_{h}^{2}} y\right)}}{2^{\frac{n-1}{2}} \Gamma\left(\frac{n-1}{2}\right)} \frac{n-1}{\sigma_{h}^{2}}
$$


To get the detection rate, we calculate the cross point $y_{c}$ of $f_{Y_{l}}(y)$ and $f_{Y_{h}}(y)$

$$
f_{Y_{l}}\left(y_{c}\right)=f_{Y_{h}}\left(y_{c}\right)
$$

By lengthy arithmetic operations,

$$
y_{c}=\log \left(\frac{\sigma_{h}^{2}}{\sigma_{l}^{2}}\right) \frac{\sigma_{h}^{2} \sigma_{l}^{2}}{\sigma_{h}^{2}-\sigma_{l}^{2}}
$$

Now we use Chebynov inequality for the estimation of error rate if the adversary uses sample variance as the feature statistic. The distance from the mean of $Y_{l}$ to the cross point $y_{c}$ is denoted as $D_{l}$

$$
D_{l}=y_{c}-\sigma_{l}^{2}
$$

Substitute (44) and (51) into (52)

$$
D_{l}=\left(\log \left(\frac{\sigma_{h}^{2}}{\sigma_{l}^{2}}\right) \frac{\sigma_{h}^{2}}{\sigma_{h}^{2}-\sigma_{l}^{2}}-1\right) \sigma_{l}^{2}
$$

Denoting $c_{l}$ as the ratio of $D_{l}$ to the standard deviation of $Y_{l}$

$$
c_{l}=\frac{D_{l}}{\sqrt{\operatorname{var}\left(Y_{l}\right)}}
$$

Substitute (45) and (53) into (54)

$$
c_{l}=\frac{\log \left(\frac{\sigma_{h}^{2}}{\sigma_{l}^{2}}\right) \frac{\sigma_{h}^{2}}{\sigma_{h}^{2}-\sigma_{l}^{2}}-1}{\sqrt{\frac{2}{n-1}}}
$$

Similarly, denoting $D_{h}$ as the distance from the mean of $Y_{h}$ to the cross point $y_{c}$,

$$
D_{h}=\sigma_{h}^{2}-y_{c}=\sigma_{h}^{2}\left(1-\log \left(\frac{\sigma_{h}^{2}}{\sigma_{l}^{2}}\right) \frac{\sigma_{l}^{2}}{\sigma_{h}^{2}-\sigma_{l}^{2}}\right)
$$

Then $c_{h}$, the ratio of $D_{h}$ and the standard deviation of $Y_{h}$, can be calculated as follows

$$
c_{h}=\frac{1-\log \left(\frac{\sigma_{h}^{2}}{\sigma_{l}^{2}}\right) \frac{\sigma_{l}^{2}}{\sigma_{h}^{2}-\sigma_{l}^{2}}}{\sqrt{\frac{2}{n-1}}}
$$

When sample size $n$ is big ( $>40$ ), we can assume that a chi square PDF is symmetrical. Thus from Chebynov inequality, we can get the error rate $e_{Y}$

$$
e_{Y} \leq \frac{\frac{1}{2 c_{l}^{2}}+\frac{1}{2 c_{h}^{2}}}{2}
$$

Thus, the detection rate $v_{Y}$ can be calculated as

$$
\begin{aligned}
v_{Y} & =1-e_{Y} \\
& \geq 1-\frac{1}{4 c_{l}^{2}}-\frac{1}{4 c_{h}^{2}}
\end{aligned}
$$


Substitute (17), (55) and (57) into (60)

$$
v_{Y} \geq 1-\frac{C}{n-1}
$$

where

$$
C_{Y}=\frac{1}{2\left(1-\frac{1}{r-1} \log r\right)^{2}}+\frac{1}{2\left(\frac{r}{r-1} \log r-1\right)^{2}}
$$

Thus, we use the lower bound of $v_{Y}$ as the estimation of the detection rate. Since that $v_{Y}$ must be greater than $50 \%$, we can get (30). The theorem is proved.

(2) In this part, we prove that $v_{Y}$ is an increasing function of $r$.

That is, we need to prove that $C_{Y}$ in (31) is a decreasing function of $r$. If in (31), $C Y 1(r)=\frac{\log r}{r-1}$ is a decreasing function and $C Y 2(r)=\frac{r}{r-1} \log r$ is an increasing function, $C_{Y}$ is a decreasing function.

We first prove that $C Y 1(r)$ is a decreasing function. Let $r=e^{x}$.

$$
\begin{aligned}
C Y 1(r) & =c y 1(x)=\frac{x}{e^{x}-1} \\
\frac{d C Y 1}{d r} & =\frac{d c y 1}{d r} \\
& =\frac{d c y 1}{d x} \frac{d x}{d r} \\
& =\frac{e^{x}-1-x e^{x}}{r\left(e^{x}-1\right)^{2}}
\end{aligned}
$$

Since $r>1, x>0$, the denominator of (66), $r\left(e^{x}-1\right)^{2}>1$. For the numerator of (66)

$$
e^{x}-1-x e^{x}=\sum_{n=1}^{\infty}\left(\frac{1}{n !}-\frac{1}{(n-1) !}\right) x^{n}
$$

Since $\frac{1}{n !}-\frac{1}{(n-1) !}<0$

$$
e^{x}-1-x e^{x}<0
$$

So $\frac{d c 1}{d r}<0, C Y 1(r)$ is a decreasing function.

Now we prove $C Y 2(r)$ is an increasing function of $r$. Let $r=e^{x}$,

$$
C Y 2(r)=\frac{r \log r}{r-1}=\operatorname{cy} 2(x)=\frac{x e^{x}}{e^{x}-1}
$$

and

$$
\frac{d C Y 2}{d r}=\frac{e^{2 x}-e^{x}-x e^{x}}{\left(r e^{x}-1\right)^{2}}
$$

Since $r>1, x>0$, and the denominator of (70), $\left(e^{x}-1\right)^{2} r>0$. For the numerator of (70),

$$
e^{2 x}-e^{x}-x e^{x}=\sum_{n=1}^{\infty}\left(\frac{2^{n}-1-n}{n !}\right) x^{n}
$$


Since $\frac{2^{n}-1-n}{n !} \geq 0$ when $n>0$

$$
e^{2 x}-e^{x}-x e^{x} \geq 0
$$

So $d C Y 2 / d r=d c y 2 / d r>0$ and $C Y 2(r)$ is an increasing function.

Thus, we have proved that $v_{Y}$ is an increasing function in terms of $r$.

\section{Appendix C}

(1) In this part, we prove Theorem 3.

Proof: A normal distribution's differential entropy can be calculated as

$$
H=\frac{\log 2 \pi \sigma^{2}+1}{2}
$$

Here we use sample variance $Y(29)$ to estimate sample entropy $\tilde{H}$.

$$
\widetilde{H}=\frac{\log 2 \pi Y+1}{2}
$$

To get sample entropy's PDF, we first derive its distribution $P(\widetilde{H}<h)$

$$
P(\widetilde{H}<h)=P\left(\frac{\log 2 \pi Y+1}{2}<h\right)=P\left(Y<\frac{e^{2 h-1}}{2 \pi}\right)
$$

Differentiating two sides of (75), we get sample entropy's PDF $f_{\widetilde{H}}(h)$

$$
f_{\widetilde{H}}(h)=f_{Y}\left(\frac{e^{2 h-1}}{2 \pi}\right)\left(\frac{e^{2 h-1}}{2 \pi}\right)^{\prime}
$$

Denote $H_{l}$ as the sample entropy of padded traffic's PIAT at the low-rate payload traffic, and $f_{H_{l}}(h)$ as $H_{l}$ 's PDF. Denote $H_{h}$ as the sample entropy of padded traffic's PIAT at the high-rate payload traffic, and $f_{H_{h}}(h)$ as $H_{h}$ 's PDF. To get the detection rate, we need to calculate the cross point $h_{c}$ of $f_{H_{l}}(h)$ and $f_{H_{h}}(h)$

$$
f_{H_{l}}\left(h_{c}\right)=f_{H_{h}}\left(h_{c}\right)
$$

By lengthy arithmetic operations

$$
h_{c}=\frac{\log \left[2 \pi \log \left(\frac{\sigma_{h}^{2}}{\sigma_{l}^{2}}\right) \frac{\sigma_{h}^{2} \sigma_{l}^{2}}{\sigma_{h}^{2}-\sigma_{l}^{2}}\right]+1}{2}
$$

For the ease of estimation, we approximate sample entropy's mean as $\frac{\log 2 \pi \sigma^{2}+1}{2}$, i.e., we assume that the entropy estimator (74) above is unbiased.

Now we use Chebynov inequality for the error rate estimation, The distance from the mean of $H_{l}$ to the cross point $h_{c}$ is denoted as $D_{l}$

$$
D_{l}=\frac{\log \left[2 \pi \log \left(\frac{\sigma_{h}^{2}}{\sigma_{l}^{2}}\right) \frac{\sigma_{h}^{2} \sigma_{l}^{2}}{\sigma_{h}^{2}-\sigma_{l}^{2}}\right]+1}{2}-\frac{\log 2 \pi \sigma_{l}^{2}+1}{2}=\frac{\log \left(\log \left(\frac{\sigma_{h}^{2}}{\sigma_{l}^{2}}\right) \frac{\sigma_{h}^{2}}{\sigma_{h}^{2}-\sigma_{l}^{2}}\right)}{2}
$$


Using Taylor expansion over (74) and by appropriate approximation, we get $\operatorname{var}(\tilde{H}) \approx \frac{1}{2 n}$ (the same result as for the histogram-based entropy estimator in [14]). Denoting $c_{l}$ as the ratio of $D_{l}$ to the standard deviation of $h_{l}$

$$
c_{l}=\frac{\log \left(\log \left(\frac{\sigma_{h}^{2}}{\sigma_{l}^{2}}\right) \frac{\sigma_{h}^{2}}{\sigma_{h}^{2}-\sigma_{l}^{2}}\right)}{2 \sqrt{\frac{1}{2 n}}}
$$

Similarly, denoting $D_{h}$ as the distance from the mean of $H_{h}$ to the cross point $h_{c}$ and $c_{h}$ as the ratio of $D_{h}$ to the standard deviation of $h_{h}$, we have

$$
\begin{aligned}
D_{h} & =\frac{\log 2 \pi \sigma_{h}^{2}+1}{2}-\frac{\log \left[2 \pi \log \left(\frac{\sigma_{h}^{2}}{\sigma_{l}^{2}}\right) \frac{\sigma_{h}^{2} \sigma_{l}^{2}}{\sigma_{h}^{2}-\sigma_{l}^{2}}\right]+1}{2}=\log \left(\frac{\sigma_{h}^{2}-\sigma_{l}^{2}}{\sigma_{l}^{2} \log \left(\frac{\sigma_{h}^{2}}{\sigma_{l}^{2}}\right)}\right) / 2 \\
c_{h} & =\log \left(\frac{\sigma_{h}^{2}-\sigma_{l}^{2}}{\sigma_{l}^{2} \log \left(\frac{\sigma_{h}^{2}}{\sigma_{l}^{2}}\right)}\right) /(2 \sqrt{1 / 2 n})
\end{aligned}
$$

So error rate $e_{\widetilde{H}}$ is calculated as

$$
e_{\widetilde{H}} \leq \frac{1}{4 c_{l}^{2}}+\frac{1}{4 c_{h}^{2}}=\frac{C_{\widetilde{H}}}{n}
$$

where

$$
C_{\widetilde{H}}=\frac{1}{2\left(\log \left(\log \left(\frac{\sigma_{h}^{2}}{\sigma_{l}^{2}}\right) \frac{\sigma_{h}^{2}}{\sigma_{h}^{2}-\sigma_{l}^{2}}\right)\right)^{2}}+\frac{1}{2\left(\log \left(\frac{\sigma_{h}^{2}-\sigma_{l}^{2}}{\sigma_{l}^{2} \log \left(\frac{\sigma_{h}^{2}}{\sigma_{l}^{2}}\right)}\right)\right)^{2}}
$$

Substitute (17) into (84), we have

$$
C_{\widetilde{H}}=\frac{1}{2\left(\log \left(\frac{r}{r-1} \log r\right)\right)^{2}}+\frac{1}{2\left(\log \left(\frac{r-1}{\log r}\right)\right)^{2}}
$$

Since

$$
v_{\tilde{H}}=1-e_{\widetilde{H}}
$$

Substitute (83) into (86), we have

$$
v_{\tilde{H}} \geq 1-\frac{C_{\widetilde{H}}}{n}
$$

In this paper, we use the lower bound of $v_{\tilde{H}}$ as the estimation of the detection rate by sample entropy. Conside that detection rate $v$ must be greater than $50 \%$ and we can get (32) in Theorem 3 . The theorem is proved.

(2) In this part we prove that $v_{\tilde{H}}$ is an increasing function in terms of $r$.

That is, we need to prove that $C_{\tilde{H}}$ is a decreasing function of $r$. If $C H 1(r)=\frac{r-1}{\log r}$ and $C H 2(r)=\frac{r}{r-1} \log r$ in (33) are increasing functions of $r, C_{\tilde{H}}$ is a decreasing function of $r$.

$C H 1(r)=1 / C Y 1(r)$ where $C Y 1(r)$ is defined in (63). Since $C Y 1(r)$ is a deceasing function of $r, C H 1(r)$ is an increasing function. $C H 2(r)=C Y 2(r)$ where $C Y 2(r)$ is defined in (69). $C Y 2(r)$ is an increasing function, so is $C H 2(r)$.

Thus $v_{\tilde{H}}$ is of $r$, where $r \geq 1$. 